\title{
Avaliação do Uso de Meta-heurísticas Baseadas no Comportamento da Natureza em Apoio a Operações de Esclarecimento por Aeronaves de Asa Móvel
}

\author{
André Muniz Yokoyama \\ Antonio R. Mury \\ Mariza Ferro \\ Bruno Schulze
}

Laboratório Nacional de Computação Científica (LNCC)

Avenida Getúlio Vargas, 333, Quitandinha, Petrópolis - Rio de Janeiro andremy, a.roberto, mariza, schulze@lncc.br

\section{RESUMO}

O principal objetivo deste trabalho é a avaliação de duas meta-heurísticas inspiradas na natureza, Algoritmos Genéticos e Colônia de Formigas, para o desenvolvimento de uma aplicação que possa gerar rotas otimizadas para aeronaves, atendendo às exigências da Marinha do Brasil. Este trabalho apresenta os métodos desenvolvidos, obedecendo a duas restrições principais: a mobilidade dos pontos de checagem e a autonomia limitada das aeronaves. Apresenta também os resultados dos testes realizados com os métodos desenvolvidos e uma avaliação de seus desempenhos.

Palavra-chave: Meta heurística; Otimização; Algoritmos Genéticos; Colônia de Formigas; TSP.

\begin{abstract}
The main objective of this work is the evaluation of two nature inspired metaheuristics, Genetic Algorithms and Ant Colony, for the development of an application that can generate optimized routes for aircraft, attending the requirements of the Brazilian Navy. This work presents the methods developed, complying with two main constraints: checkpoints mobility and limited aircraft autonomy. It also presents the results of tests performed with the methods developed and an evaluation of their performances.
\end{abstract}

Keywords: Meta-heuristic; Optimization; Genetic Algorithms; Ant Colony; TSP.

\section{Como Citar:}

YOKOYAMA, André Muniz; MURY, Antonio R.; FERRO, Mariza; SCHULZE, Bruno. Avaliação do Uso de Meta-heurísticas Baseadas no Comportamento da Natureza em Apoio a Operações de Esclarecimento por Aeronaves de Asa Móvel. In: SIMPÓSIO DE PESQUISA OPERACIONAL E LOGÍSTICA DA MARINHA, 19., 2019, Rio de Janeiro, RJ. Anais [... ]. Rio de Janeiro: Centro de Análises de Sistemas Navais, 2019. 


\section{INTRODUÇÃO}

A Marinha do Brasil em 2004 apresentou pela primeira vez o termo Amazônia Azul, por meio do então Comandante da Marinha Roberto Guimarães Carvalho, com o intuito de divulgar, despertar o interesse e esclarecer para a população esta riqueza que existe em nossa vasta costa [1].

A área correspondente à Amazônia Azul, que consiste na soma das áreas correspondentes a Zona Econômica Exclusiva e a Plataforma Continental, chega a 4,5 milhões de quilômetros quadrados. Este território marítimo, ao contrário do que é comumente divulgado, não contém apenas as nossas reservas de petróleo e gás natural, ela contém também todas as riquezas minerais do subsolo; em sua superfície, as nossas vias marítimas; e uma inumerável diversidade biológica [2, 3].

Porém, para poder exercer os seus direitos, o Brasil deve ser capaz de exercer o seu poder marítimo, que resulta da integração dos recursos de que dispõe a Nação para a utilização do mar e das águas interiores, quer como instrumento de ação política e militar, quer como fator de desenvolvimento econômico e social. O Poder Naval, parcela militar integrante do Poder Marítimo, por meio da coordenação da Marinha do Brasil, deve estar capacitado a atuar militarmente no mar, em águas interiores e em certas áreas terrestres limitadas, de interesse para as operações navais, incluindo o espaço aéreo sobrejacente visando conquistar e manter os objetivos nacionais [4]. E neste caso, lembrando as palavras de Rui Barbosa: - "Uma nação que confia em seus direitos, em vez de confiar em seus soldados, engana-se a si mesma e prepara a sua própria queda".

O trabalho aqui apresentado trata de uma das missões de uma força naval e seus meios, que consiste na busca e esclarecimento do território marítimo brasileiro. Esta operação de busca e esclarecimento tem por finalidade contribuir para a vigilância no nível operacional e estratégico, realizando a identificação dos meios que se encontram na área, e se necessário a neutralização de meios hostis.

Neste trabalho é tratado um dos aspectos das operações de esclarecimento aéreo, realizado no âmbito de uma força naval, operando em uma área marítima. Estas operações são levadas a cabo por helicópteros de esclarecimento e ataque embarcados nos navios da Marinha do Brasil. O objetivo é determinar o melhor trajeto que esta aeronave de asa móvel deverá percorrer, de forma a poder esclarecer o maior número de alvos em uma determinada área.

Este problema se assemelha ao problema do caixeiro viajante, "Traveling Salesman Problem"(TSP), no entanto, possui duas diferenças significativas em relação ao TSP clássico: os pontos de destino se movimentam em direções e velocidades diversas, assim como a necessidade da aeronave retornar ao seu navio-mãe (navio onde a aeronave esta embarcada), dentro da sua autonomia de voo.

Para isso foram elaborados métodos baseados em duas meta-heurísticas inspiradas na natureza: Algoritmos Genético (AG) e Otimização por Colônia de Formigas (OCF). Este trabalho avalia o uso dessas meta-heurísticas na busca do percurso que maximiza o número de alvos esclarecidos, em função da autonomia da aeronave, minimizando a distância percorrida.

\subsection{Trabalhos Relacionados}


O uso de meta-heurísticas inspiradas na natureza, em especial o OCF e AG, para solução de problemas do tipo NP-hard, como por exemplo o TSP, têm apresentado bons resultados [5, 6]. Por este motivo, optou-se por avaliar o uso destas duas meta-heurísticas, OCF e AG, uma vez que o problema tratado se assemelha ao TSP. Após uma extensa revisão da literatura, foram encontrados trabalhos que fazem adaptações desses métodos para o problema do TSP, como [7] para o OCF and [8] para o AG. No entanto, não foram encontrados estudos que adaptassem esses métodos ao problema abordado neste trabalho.

Como mencionado, com base no problema descrito, foram elaborados e avaliados os métodos AG e OCF na busca do percurso que maximiza o número de alvos esclarecidos, em função da autonomia da aeronave, minimizando a distância percorrida. A metodologia para este trabalho é apresentada na Seção 2. O detalhamento dos métodos propostos e os resultados experimentais são apresentados nas Seções 3 e 4 . Finalmente na Seção 5 são apresentadas as considerações finais.

\section{METODOLOGIA E PROPOSTA}

Esta seção apresenta a metodologia aplicada nos experimentos, as funções propostas para cada método e a descrição dos experimentos. A metodologia adotada neste trabalho está dividida em duas etapas principais: A primeira etapa concentra-se no desenvolvimento de métodos baseados no AG e na OCF para a solução do problema proposto, levando em consideração apenas a mobilidade dos alvos. A segunda etapa adapta a aplicação criado na etapa 1 para incluir uma nova restrição: o limite de autonomia da aeronave. Isso requer a inclusão de um novo objetivo para o problema e a maximização do número de alvos identificados, uma vez que, dado o alcance limitado da aeronave, pode não ser possível identificar todos os alvos.

Para cada etapa é proposto um conjunto de testes que possuem características em comum e também parâmetros específicos, tanto para AG como para OCF. Porém, comum a todos os testes serão usados dois conjuntos de dados diferentes denominados de 'C1' e 'C2'. Esses conjuntos de dados são gerados aleatoriamente, mas seguem certas restrições para torná-los o mais próximo possível de um cenário da vida real. Eles incluem os alvos dispostos de forma aleatória porém, respeitando uma distância mínima de 15 milhas náuticas, que equivale aproximadamente à distância da linha do horizonte, onde os alvos poderiam ser visualmente identificados pelo próprio navio-mãe, e respeitando o limite máximo de velocidade de 32 nós (milhas náuticas por hora), equivalente a velocidade média de ataque de navios militares. O sistema de coordenadas utilizado foi o plano cartesiano, mas a aplicação pode usar a coordenada esférica ou geográfica, se necessário. O número de destinos para cada conjunto de dados foi definido como 20.

Os específicos para AG e OCF são: baseados na literatura e nos testes iniciais com o TSP clássico, concluiu-se que o tamanho populacional para o AG de 100 indivíduos seria o ideal para uma série de 20 alvos. Para OCF, ele foi definido como igual ao número de destinos nos conjuntos de dados (20). Em relação ao número de indivíduos a serem preservados ou elitismo para AG, optamos por usar o elitismo para preservar o melhor indivíduo da população recém-gerada e preservar os dois melhores indivíduos em cada geração. Para AG, a probabilidade de cruzamento $=80 \%$ e probabilidade de mutação $=1 \%$. Número máximo de gerações: para o AG avaliou-se a curva de evolução [9] da qualidade das soluções em relação ao número de gerações e não se observou qualquer melhora nos resultados (em 99\% casos) além de 1000 gerações. Para ACO = 200. 


\section{MÉTODOS PROPOSTOS E RESULTADOS- ETAPA 1}

Esta primeira etapa é focada no desenvolvimento de métodos baseados nas metaheurísticas OCF e AG. As funções para cada método foram desenvolvidas com base nas funções clássicas. Mas, para atender aos requerimentos e restrições do problema abordado neste trabalho, propomos nossos próprios métodos. Aqui apresentamos apenas as funções mais importantes implementadas, devido às limitações de espaço. O conjunto completo de funções está em [9].

Nesta etapa é considerada apenas a mobilidade dos pontos (alvos) a serem identificados. Para o TSP clássico, as distâncias entre os pontos a serem visitados são previamente conhecidas ou são calculadas na inicialização da aplicação e essas distâncias são armazenadas em uma matriz de distâncias. No entanto, devido ao fato de que no problema abordado os alvos estão se movendo, a matriz de distância não pode ser determinada no início da aplicação. A distância entre cada alvo varia de acordo com sua ordem na rota, bem como a ordem dos alvos anteriores. Os métodos propostos e resultados obtidos sobre os dois conjuntos de dados (C1 e C2), são apresentados a seguir.

\section{Funções Propostas para o OCF}

A função de exploração é responsável pela construção das rotas de cada formiga. A montagem da rota é sempre iniciada pelo alvo 0, isto é, o navio-mãe. A partir dai a seleção dos próximos passos é realizado por um método de roleta simples onde a probabilidade de um alvo ser escolhido é diretamente proporcional a quantidade de feromônio na aresta entre o alvo atual e o alvo candidato e inversamente proporcional a distância entre eles, dada pela Equação 1

$$
P_{j}= \begin{cases}\frac{\tau_{i j}^{\alpha} \cdot \delta_{i j}^{\beta}}{\sum_{k \in N} \tau_{i k}^{\alpha} \cdot \delta_{i k}^{\beta}} & \text { se } j \in \mathrm{N} \\ 0 & \text { se } j \notin \mathrm{N}\end{cases}
$$

onde:

- $i$ é o alvo atual

- $j$ o alvo candidato

- $\tau_{i j}$ a quantidade de feromônio entre os alvos $i$ e $j$

- $\delta_{i j}=\frac{1}{D_{i j}}$

- $D_{i j}$ a distância entre os alvos $i$ e $j$

- $\mathrm{N}$ é o conjunto de todos os alvos que ainda não tenham sido selecionados

- $\alpha$ e $\beta$ são pesos que definem o grau de relevância dada a cada parcela

Para os testes foram definidos $\alpha=1$ e $\beta=2$.

Esta função também é responsável por invocar a função de depósito de feromônio ao final da construção de cada rota e a função de evaporação de feromônios [9] ao final de cada iteração. A função de inicialização das trilhas é responsável pela inicialização 
das possíveis trilhas adicionando feromônio às arestas. Para os testes realizados todas as arestas foram iniciadas com a mesma quantidade de feromônio $\left(\tau_{i j}=0,1\right)$.

Nesta etapa o método baseado em Otimização por Colônia de Formigas desenvolvido foi denominado ACO.

\section{Funções Propostas para os métodos baseados em Algorítimo Genético}

A primeira função, Geração de indivíduos com igual probabilidade de escolha dos alvos gera uma permutação dos alvos disponíveis (cromossomo) de forma aleatória, sem nenhum viés. Porém, é sempre iniciada pelo alvo 0, isto é, o navio-mãe. Os testes realizados nessa etapa que utilizaram essa função foram denominados GA. Para a segunda função, Geração de indivíduos com probabilidade de escolha dos alvos proporcional a distância a montagem do cromossomo é sempre iniciada pelo alvo 0, isto é, o navio-mãe. A partir daí a seleção dos próximos alelos é realizada por um método de roleta simples, onde a probabilidade de um alvo ser escolhido é inversamente proporcional a distância entre o alvo atual e o alvo candidato, dada pela Equação 2

$$
P_{j}= \begin{cases}\frac{\delta_{i j}^{2}}{\sum_{k \in N} \delta_{i k}^{2}} & \text { se } j \in \mathrm{N} \\ 0 & \text { se } j \notin \mathrm{N}\end{cases}
$$

onde:

- $i$ é o alvo atual

- $j$ o alvo candidato

- $\delta_{i j}=\frac{1}{d_{i j}}$

- $d_{i j}$ a distância entre os alvos $i$ e $j$

- $\mathrm{N}$ é o conjunto de todos os alvos que ainda não tenham sido selecionados

GA-2.

Os testes realizados nessa etapa que utilizaram essa função foram denominados

A função de mutação implementada nesta etapa seleciona aleatoriamente dois alelos distintos quaisquer do cromossomo do indivíduo, com a exceção do primeiro alelo da sequência, e troca as suas respectivas posições.

A Função de aptidão é responsável por avaliar a aptidão de cada indivíduo da população. A aptidão é inversamente proporcional à distância percorrida na rota representada pelo indivíduo e é obtida pela Equação 3 .

$$
f_{i}=d_{\min }+d_{\max }-d_{i}
$$

onde $d_{\min }$ é a menor distância observada na população avaliada, $d_{\max }$ a maior distância observada e $d_{i}$ a distância do i-ésimo indivíduo.

Funções para Crossover e algumas outras funções não apresentadas aqui são detalhadas em [9].

Resultados para os conjuntos de dados C1 e C2: 
Na Tabela 1 estão relacionados o menor e maior erro obtidos por cada método (GA, GA-2 e ACO), a média obtida nos testes e o percentual de resultados observados acima da tolerância de 20\%, para os conjuntos de dados C1 e C2. Os melhores valores estão representados em negrito.

\begin{tabular}{|l|c|c|c|c|c|c|}
\cline { 2 - 7 } \multicolumn{1}{c|}{} & \multicolumn{3}{c|}{ C1 } & \multicolumn{3}{c|}{ C2 } \\
\cline { 2 - 7 } \multicolumn{1}{c|}{} & GA & GA-2 & ACO & GA & GA-2 & ACO \\
\hline Menor erro (\%) & $\mathbf{0}$ & 3.07 & 0.61 & $\mathbf{0}$ & $\mathbf{0}$ & 1.54 \\
\hline Média (\%) & 12.77 & $\mathbf{5 . 4 9}$ & 17.01 & 15.45 & 10.80 & $\mathbf{1 0 . 7 5}$ \\
\hline Maior erro (\%) & 49.85 & $\mathbf{2 8 . 9 0}$ & 31.41 & 55.19 & 31.74 & $\mathbf{2 2 . 5 9}$ \\
\hline Erros acima de 20\% & 25.4 & $\mathbf{0 . 0 1}$ & 25.8 & 29.7 & 9.2 & $\mathbf{0 . 6}$ \\
\hline Tempo médio de execução & 18 seg & 18 seg & $\mathbf{1 ~ s e c}$ & 18 seg & 18 seg & $\mathbf{1 ~ s e g}$ \\
\hline
\end{tabular}

Tabela 1: Resultados para os conjuntos de dados C1 e C2

Observando a Figura 1 e o primeiro conjunto de colunas podemos observar os resultados para o conjunto de dados C1. Erros para o GA-2 estão concentrados principalmente na faixa de $4 \%$ (mais de $60 \%$ de ocorrências). Embora para GA os picos de concentração fossem semelhantes como no GA-2, a concentração foi muito menor, e os resultados foram muito mais distribuídos sobre as bandas de erro observadas. Para o ACO, os resultados foram distribuídos entre faixas de $10 \%$ a $21 \%$.

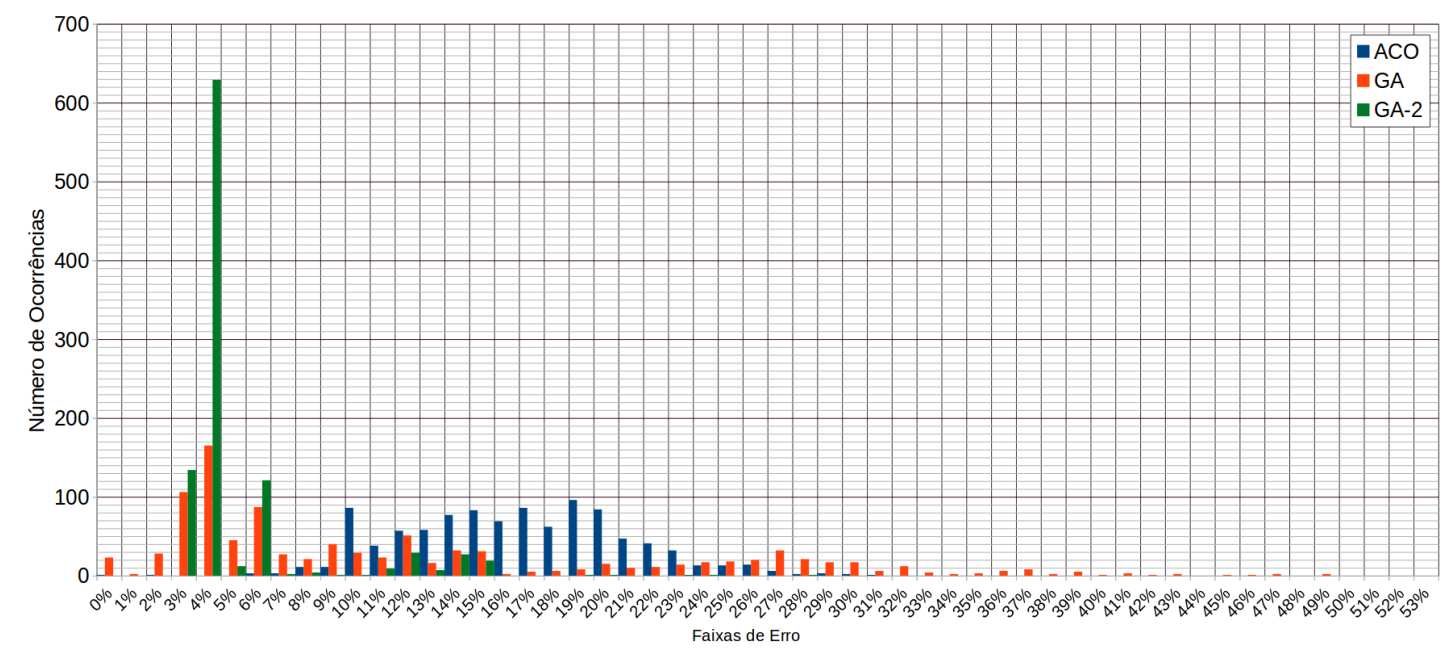

Figura 1: Gráfico combinado dos histogramas dos resultados para o conjuntos de dados $\mathrm{C} 1$

Nas colunas finais da Tabela 1 e na Figura 2 são apresentados os resultados para o conjunto de dados C2. Para os três métodos, a maior concentração de resultados está entre as bandas de erro de $2 \%$ e $20 \%$. Para ACO, os principais pontos de concentração estão em 10 e $6 \%$ de bandas de erro. Para o GA-2, a maior concentração de resultados está nas bandas de 2, 5, 6 e 10\%. Esses resultados são distribuídos por um intervalo muito mais longo do que o ACO, variando de 0 a $31 \%$, implicando em uma qualidade de resultados inferior à do ACO. A GA obteve a pior qualidade geral dos resultados, que são distribuídos por um intervalo muito mais longo do que para outros métodos (até o intervalo de $55 \%$ ).

Para ambos os conjuntos de dados, embora os tempos de execução para GA e GA-2 tenham sido consideravelmente mais longos (cerca de 18 segundos) do que para 


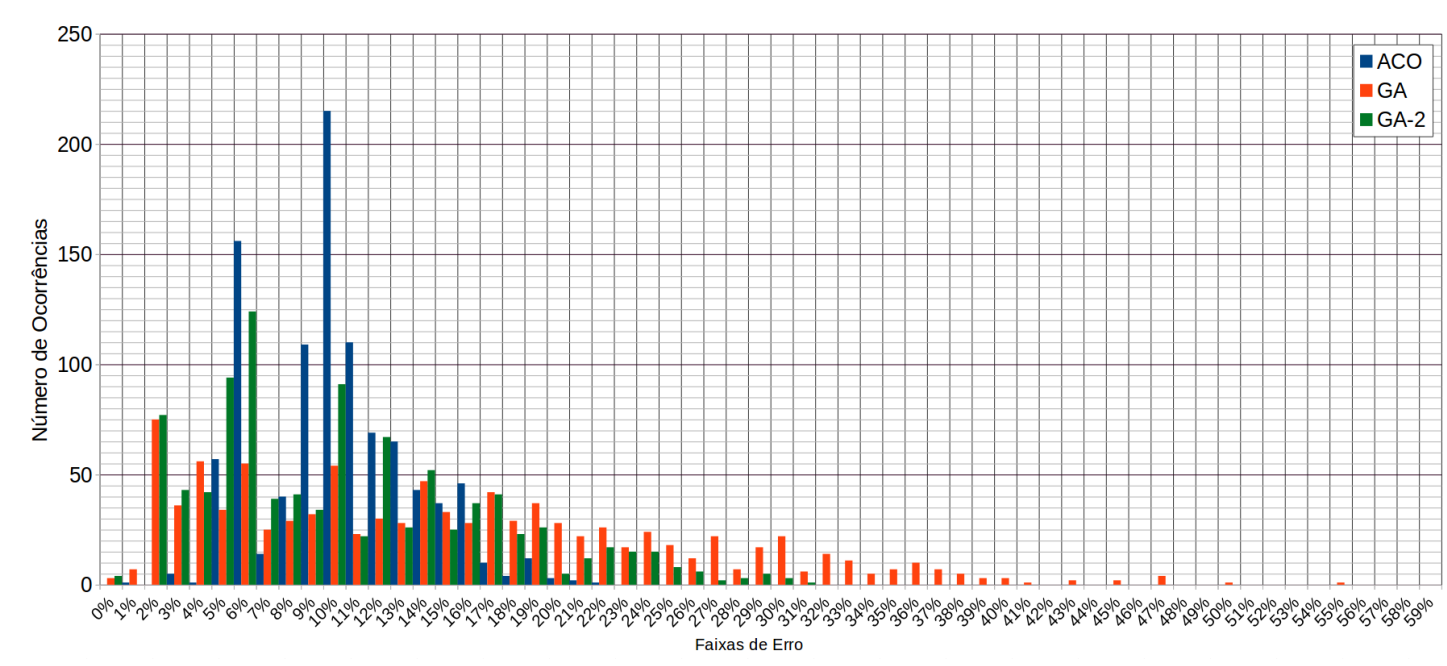

Figura 2: Gráfico combinado dos histogramas dos resultados para o conjuntos de dados $\mathrm{C} 2$

ACO (um segundo), os tempos para os três métodos foram considerados muito bons. Estes tempos permitem, por exemplo, múltiplas execuções dos métodos durante o briefing da missão, ou até executar o aplicativo durante o vôo da aeronave, caso fosse necessário traçar uma nova rota. Estes resultados apontam para a viabilidade dos métodos propostos e implementados nesta etapa.

\section{MÉTODOS PROPOSTOS E RESULTADOS- ETAPA 2}

Nesta etapa é incluída mais uma restrição ao problema, o limite de autonomia da aeronave. A inclusão desta nova restrição implica que nem todos os alvos designados possam ser esclarecidos sem que a aeronave tenha que retornar ao navio-mãe para reabastecimento. Assim, faz-se necessário a inclusão de um novo objetivo: a maximização do número de alvos esclarecidos em função do limite de autonomia, pois apenas a minimização da rota não produziria soluções aceitáveis. Já que não há a necessidade de esclarecer todos os alvos, a menor rota seria esclarecer apenas o alvo que apresentasse a menor distância total (somatório das distâncias de ida e volta) em relação ao navio-mãe. A seguir serão descritas, devido a limitação de espaço, algumas das adaptações feitas nos algoritmos utilizados na Etapa 1 da pesquisa. O conjunto completo de funções adaptadas e implementadas está disponível em [9]. Estas adaptações incluem modificações de funções utilizadas anteriormente e a inclusão de novas funções, para atender a limitação de autonomia de voo da aeronave.

\section{Novas Funções Propostas para o ACO:}

A Função de depósito de feromônio foi adaptada para adicionar uma quantidade de feromônio que é inversamente proporcional ao comprimento de cada aresta $(i, j)$ dada pela Equação 4

$$
\Delta \tau_{i j}^{k}= \begin{cases}\frac{C}{d_{i j}} & \text { se a aresta }(i, j) \text { fizer parte da rota da formiga } \mathrm{k} \\ 0 & \text { para as demais }\end{cases}
$$

$C$ é uma constante e $d_{i j}$ a distância da aresta $(i, j)$ percorrida pela formiga $k$. 
Para a função de exploração foram utilizadas duas versões. Para ambas a construção da rota é realizada de forma similar ao que foi visto na etapa 1 e dada pela mesma Equação 1. Na primeira versão utilizada nesta fase, após a seleção de cada alvo (com exceção do navio mãe), a distância total da rota é avaliada. Caso essa distância seja maior do que o limite máximo de autonomia da aeronave, o alvo recém selecionado é descartado, permanecendo apenas os alvos selecionados anteriormente, e o processo de construção da rota é terminado. Se a distância for menor que o limite de autonomia, o processo de construção da rota continua até que a distância limite seja ultrapassada, ou todos os alvos sejam selecionados. Considera-se como distância total da rota a distância até o último alvo selecionado mais a distância de retorno ao navio-mãe.

Durante a construção da rota também é realizado o depósito de feromônio na aresta entre o alvo selecionado anteriormente e o alvo recém selecionado por meio da função de depósito de feromônio. Ao final da construção da rota, se esta possuir ao menos um alvo válido, isto é, qualquer alvo diferente do "0" (navio-mãe), ela é então armazenada até que se tenha o número total de rotas definido para cada iteração. Esse número é igual ao número de alvos no conjunto de dados.

Em seguida é aplicada as função de avaliação sobre o conjunto de rotas gerado. Após a avaliação das rotas e aplicado a função de evaporação de feromônio sobre todas as arestas. Este processo é repetido a cada iteração. Para os testes realizados o número máximo de iterações por execução foi definido como 200.

Os testes que foram realizados com essa versão da função de exploração foram denominados $\mathrm{ACO}$

Na segunda versão da função de exploração utilizada, não é realizada a checagem se a rota continua válida após a inserção de cada novo alvo, como na primeira versão citada acima. Como na etapa 1 , novos alvos são adicionados à rota até que todos os alvos do conjunto de dados tenham sido adicionados. Após a construção da rota, com todos os alvos disponíveis, inicia-se o processo de sua avaliação. Este é um processo iterativo onde a primeira iteração consiste em avaliar a distância total da rota com todos os passos, isto é, todos os alvos diferentes de " 0 ". A cada nova iteração o número de passo é decrescido de 1, ignorando-se os passos ao final da rota. Este processo segue até que o único alvo restante seja o "0".

A cada iteração, caso a distância total da rota até o último alvo avaliado ultrapasse o limite de autonomia da aeronave, este alvo da rota é substituído por "0". Porém, se a distância total for menor ou igual a autonomia, a função de depósito de feromônio é aplicada sobre as arestas até o último alvo avaliado na iteração. Desta forma, a porção inválida da rota é excluída e a parte válida é reforçada. Ao final deste processo a rota é armazenada e o processo de criação de uma nova rota é iniciado. Este processo é repetido até que o número de rotas armazenadas seja igual ao número total de alvos como na primeira versão citada acima. São então aplicadas as funções de avaliação e de evaporação de feromônio. Este processo é repetido a cada iteração até atingir o número máximo de iterações. Para os testes realizados o número máximo de iterações por execução foi definido como 200.

Os testes que foram realizados com essa segunda versão da função de exploração foram denominados ACO-2.

Para a função avaliação, diferente da etapa 1 onde as rotas eram avaliadas apenas 
pela distância total, e quanto menor a rota melhor a solução, a avaliação nesta etapa é feita sobre distância da rota e o número de alvos esclarecidos na rota de forma que quanto maior o número de alvos melhor a rota, dada pela Equação 5 .

$$
f_{i}=\left(\left(l s_{\min }+l s_{\max }\right)-l s_{i}\right) *\left(\frac{s_{i}}{S}\right)
$$

onde:

- $l s_{i}$ é a distância média por passos da i-ésima rota definida por $l s_{i}=\frac{l_{i}}{s_{i}}$

- $l s_{\text {min }}$ é o menor valor de $l s_{i}$ no conjunto de rotas avaliado

- $l s_{\max }$ é o maior valor de $l s_{i}$ no conjunto de rotas avaliado

- $l_{i}$ é a distância total da i-ésima rota

- $s_{i}$ é o número de alvos da i-ésima rota

- $S$ é o número total de alvos do o conjunto de dados de entrada

\section{Novas Funções Propostas para os métodos baseados em Algorítimo Genético}

Devido ao fato de que os indivíduos pais nesta etapa podem ter quantidades diferentes de alvos, a Função Crossover precisa de algumas adaptações. Para Função Fitness, a aptidão (definida pela Equação 5) é dada pela distância da rota representada por cada indivíduo e pelo número de alvos exclarecidos, de forma que quanto menor a rota e maior o número de alvos, melhor a aptidão.

A função de mutação implementada nesta função é similar a usada na etapa 1 , com dois alelos selecionados para serem permutados, sendo o primeiro selecionado entre os alelos do indivíduo sobre o qual a função esta sendo aplicada, com a exceção do primeiro alelo da sequência; e em seguida é criado um conjunto de alelos candidatos. Este conjunto de candidatos inclui todos os alvos do conjunto de dados de entrada com exceção dos alvo 0 e do alvo representado pelo primeiro alelo selecionado para mutação.

O segundo alelo é selecionado do conjunto de candidatos, caso este alelo faça parte da sequência do indivíduo, a posição dos dois alelos são permutados. Caso contrario, o primeiro alelo é substituído pelo segundo. Desta forma é garantido que nenhum elemento na sequência se repita e também permite que elementos que não faziam parte da sequência inicial sejam incluídos.

Esta função também decide de forma aleatória se a operação de mutação ocorrerá ou não, podendo inclusive ocorrer múltiplas vezes.

As funções para Geração de individuos, Seleção e Evolução são apresentadas em $[9]$.

\section{Resultados para os conjuntos de dados C1 e C2:}

A análise foi feita com base no percentual de erro relacionado à melhor solução encontrada para cada conjunto de dados ( $\mathrm{C} 1$ e $\mathrm{C} 2)$. O erro é calculado sobre o valor normalizado da aptidão, onde a maior aptidão foi considerado como $100 \%$ e é dado por $e_{i}=100-a_{i}$ onde $a_{i}$ é a aptidão normalizada valor da solução avaliada. 
Na Tabela 2 estão relacionados os resultados para os conjuntos de dados C1 e C2. Nesta tabela podemos observar que todos os métodos usando C1 obtiveram soluções com erro $0 \%$. O método ACO-2 obteve o menor erro médio (0,06\%). Os erros obtidos pela ACO e ACO-2 ficaram abaixo de $6 \%$, enquanto para o GA e GA-2 ficaram acima de $45 \%$. Além disso, apenas os métodos ACO e ACO-2 apresentaram todos os resultados abaixo da tolerância de $20 \%$ de erro.

\begin{tabular}{|l|c|c|c|c|c|c|c|c|}
\cline { 2 - 10 } \multicolumn{1}{c|}{} & \multicolumn{4}{c|}{ C1 } & \multicolumn{4}{c|}{ C2 } \\
\cline { 2 - 10 } \multicolumn{1}{c|}{} & GA & GA-2 & ACO & ACO2 & GA & GA-2 & ACO & ACO2 \\
\hline Menor erro (\%) & $\mathbf{0}$ & $\mathbf{0}$ & $\mathbf{0}$ & $\mathbf{0}$ & $\mathbf{0}$ & $\mathbf{0}$ & $\mathbf{0}$ & $\mathbf{0}$ \\
\hline Média (\%) & 12.19 & 4.87 & 0.24 & $\mathbf{0 . 0 6}$ & 16.83 & 3.15 & $\mathbf{0 . 0 2 6}$ & 0.032 \\
\hline Maior erro (\%) & 50.82 & 46.97 & $\mathbf{5 . 1 1}$ & 5.47 & 52.15 & 38.39 & 6.45 & $\mathbf{5 . 9 8}$ \\
\hline Erros acima de 20\% & 24.5 & 0.3 & $\mathbf{0}$ & $\mathbf{0}$ & 35.5 & 0.7 & $\mathbf{0}$ & $\mathbf{0}$ \\
\hline Tempo médio de execução & 5 seg. & 5 seg. & $\mathbf{1} \mathbf{~ s e g . ~}$ & $\mathbf{1}$ seg. & 5 seg. & 5 seg. & $\mathbf{1 ~ s e g . ~}$ & $\mathbf{1}$ seg. \\
\hline
\end{tabular}

Tabela 2: Resultados para os conjuntos de dados C1 e C2 - Step 2

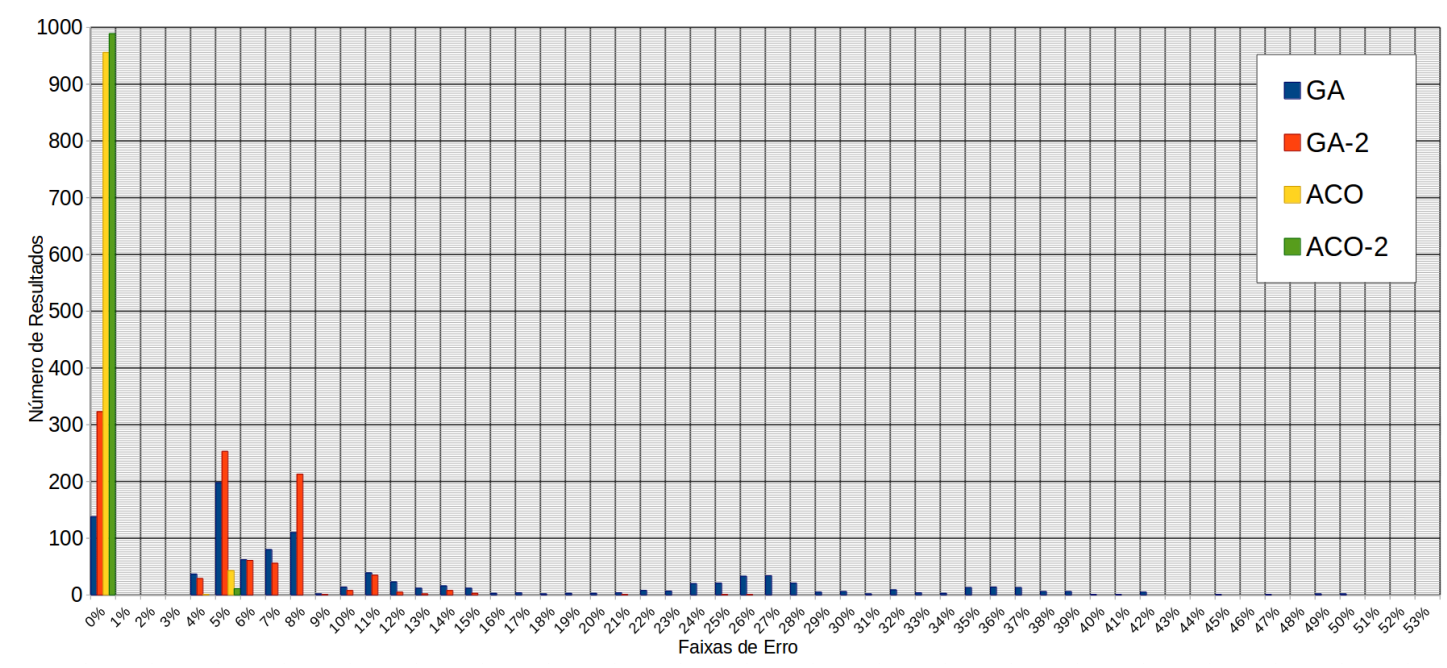

Figura 3: Gráfico combinado dos histogramas dos resultados para o conjuntos de dados $\mathrm{C} 1$

No gráfico da Figura 3 são apresentados os resultados para o conjunto de dados C1. Pode-se observar que o ACO-2 apresentou o ponto de concentração na faixa de $0 \%$ de erro (98,9\% dos resultados), apresentando a melhor qualidade geral dos resultados. Ele é seguido por ACO, com 95,6\% e GA-2 com 32,3\% e GA com 13,8\% dos resultados no intervalo de $0 \%$ de erro.

Os resultados do conjunto de dados C2 (Figura 4 ) são semelhantes a C1. ACO e ACO-2 têm uma superioridade da qualidade geral das soluções, com $98,4 \%$ e $98,2 \%$ dos resultados no intervalo $0 \%$, respectivamente, e para ambos o erro não excedeu $6 \%$. Embora o GA-2 tenha obtido uma boa quantidade de resultados na faixa de erro de $0 \%$ ( $66 \%$ dos resultados), os resultados foram distribuídos por um maior intervalo de erros, atingindo $23 \%$ de erro. Assim, apresentando uma qualidade geral inferior a ACO e ACO-2, mas ainda sendo considerada boa. O GA obteve uma pequena quantidade de resultados na faixa de erro de $0 \%$, com um pico considerável na faixa de erro de $15 \%$, resultando em uma qualidade geral muito baixa. 


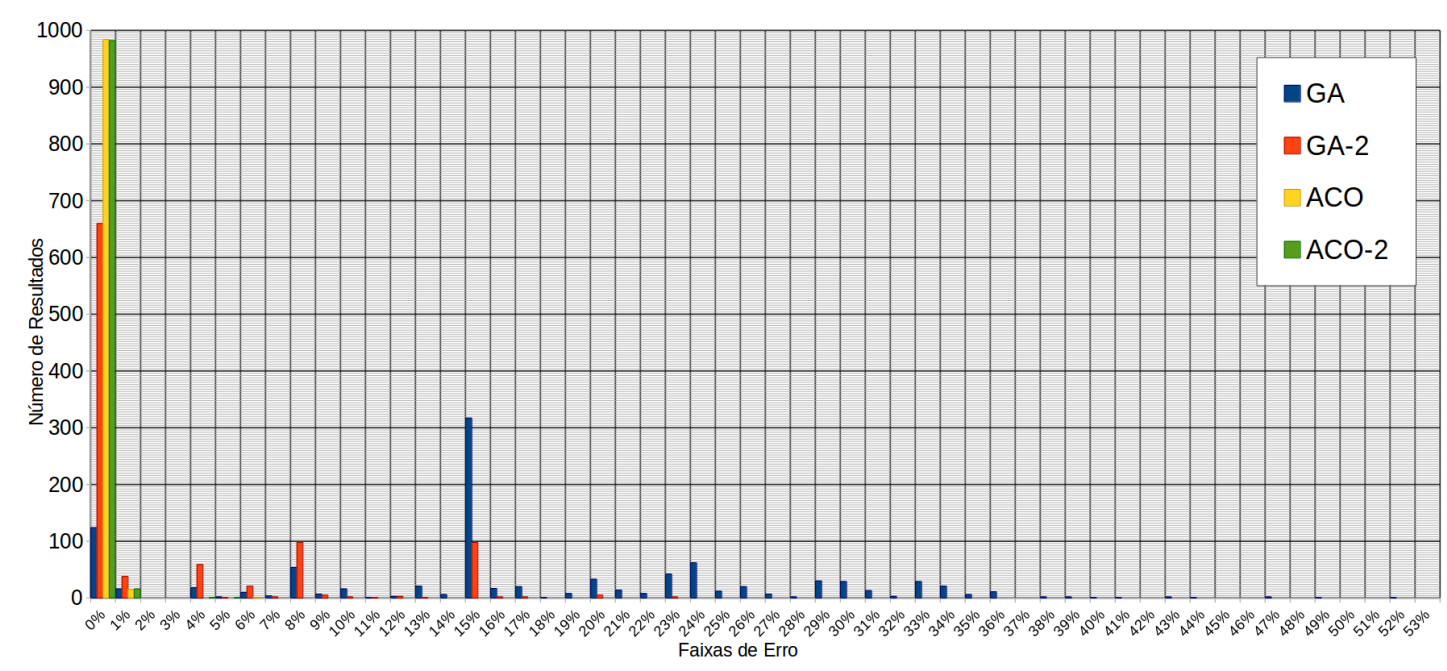

Figura 4: Gráfico combinado dos histogramas dos resultados para o conjuntos de dados $\mathrm{C} 2$

Para ambos, C1 e C2 (Tabela 2), os tempos de execução foram em média de 1 segundo para ACO e ACO-2 e aproximadamente 5 segundos para GA e GA-2. Em geral, esses tempos foram considerados muito bons para os quatro métodos, permitindo, por exemplo, a execução múltipla dos métodos durante o briefing da missão, ou até mesmo para executar o aplicativo em vôo, se uma nova rota for necessária.

\section{CONCLUSÃO}

Analisando os resultados obtidos com as duas meta-heurísticas, podemos concluir que os métodos propostos e implementados são boas abordagens para resolver o problema de reconhecimento aéreo de alvos. Embora ambas as heurísticas tenham apresentado resultados satisfatórios, o uso dos métodos baseados na otimização de colônias de formigas é o mais adequado para o problema abordado neste trabalho. Não só o ACO apresentaram os melhores resultados globais, mas também o melhor tempo de execução. No entanto, é importante notar que, embora essa meta-heurística tenha sido usada com bastante sucesso para otimizar soluções para problemas combinatórios, elas tendem a sofrer com " armadilhas " de ótimos locais dependendo do tamanho dos problemas. Além disso, o tempo de execução tende a aumentar devido ao tamanho do problema. Os experimentos realizados neste trabalho foram baseados em 20 alvos, mas os testes preliminares suportam a análise apresentada aqui para até 60 alvos.

Como trabalhos futuros são considerados adaptar os métodos desenvolvidos neste trabalho para incluir três novas restrições (importância, identificação obrigatória e priorização de alvos) e a otimização do algoritmo através do uso de computação distribuída.

\section{REFERÊNCIAS BIBLIOGRÁFICAS}

[1] CARvalho, R. Guimaraes de. A outra amazonia publicado em http://www1.folha.uol.com.br/fsp/opiniao/fz2502200409.htm. 02 2004. Disponível em: <http://www1.folha.uol.com.br/fsp/opiniao/fz2502200409.htm>. 2 
[2] COSTA, S. L. M. Amazonia azul, novas fronteiras, esg - escola superior de guerra, rio de janeiro, monografia, 2013. 01-12 2013. Disponível em: <http://www1.folha.uol.com.br/fsp/opiniao/fz2502200409.htm>. 2

[3] WIESEBRon, M. Amazonia azul: Pensando a defesa do territorio maritimo brasileiro, austral: Revista brasileira de estrategia e relacoes internacionais e-issn 22386912 issn 2238-6262, v.2, n.3, jan-jul 2013, p.107-131. 01-07 2013. Disponível em: <http://www1.folha.uol.com.br/fsp/opiniao/fz2502200409.htm>. 2

[4] DEFESA, M. da. Doutrina militar de defesa, md51-m-04, secretaria de politica, estrategia e assuntos internacionais, brasilia, df, brasil, 2007. 01-12 2007. Disponível em: $<$ http://www1.folha.uol.com.br/fsp/opiniao/fz2502200409.htm>. 2

[5] YANG, J. et al. An ant colony optimization method for generalized tsp problem. Progress in Natural Science, v. 18, n. 11, p. 1417 - 1422, 2008. ISSN 1002-0071. Disponível em: <http://www.sciencedirect.com/science/article/pii/S1002007108002736>. 3

[6] CHATTERJEE, S.; CARRERA, C.; LYNCH, L. Genetic algorithms and traveling salesman problems. European Journal of Operational Research, v. 93, n. 3, p. 490510, 1996. Disponível em: <http://www.scopus.com/inward/record.url?eid=2-s2.00030247388\&partnerID=40\&md5=0f6e75c35b59dc9b8ba26387597b0f54> . 3

[7] LI, B.; WANG, L.; SONG, W. Ant colony optimization for the ts problem based on ants with memory. In: IEEE. 4th International Conference on Natural Computation. [S.1.], 2008. 3

[8] HASSAN, M. R.; HASAN, M. K.; HASHEM, M. M. A. An improved acs algorithm for the solutions of larger tsp problems. CoRR, abs/1304.3763, 2013. Disponível em: $<$ http://arxiv.org/abs/1304.3763>. 3

[9] YOKOYAMA, A. M. Avaliação do Uso de Meta-heurísticas Baseadas no Comportamento da Natureza em Apoio a Operações de Esclarecimento por Aeronaves de Asa Móvel. Dissertação (Mestrado) - Laboratório Nacional de Computaccão Científica, 2016. http://comcidis.lncc.br/downloads/dissertacao/mestrado/tIw2DDb3WqS. pdf. 3, 4, 5, 7, 9 